\title{
Article
}

\section{Enhanced mechanical, thermal and flame retardant properties by combining graphene nanosheets and metal hydroxide nanorods for Acrylonitrile- Butadiene-Styrene copolymer composite}

Hong, Ningning, Zhan, Jing, Wang, Xin, Stec, Anna A, Hull, T Richard, Ge, Hua, Xing, Weiyi, Song, Lei and Hu, Yuan

Available at http://clok.uclan.ac.uk/11656/

Hong, Ningning, Zhan, Jing, Wang, Xin, Stec, Anna A ORCID: 0000-0002-68610468, Hull, T Richard ORCID: 0000-0002-7970-4208, Ge, Hua, Xing, Weiyi, Song, Lei and Hu, Yuan (2014) Enhanced mechanical, thermal and flame retardant properties by combining graphene nanosheets and metal hydroxide nanorods for Acrylonitrile-Butadiene-Styrene copolymer composite.

Composites Part A: Applied Science and Manufacturing, 64 . pp. 203-210. ISSN $1359835 X$

It is advisable to refer to the publisher's version if you intend to cite from the work. http://dx.doi.org/10.1016/j.compositesa.2014.04.015

For more information about UCLan's research in this area go to http://www.uclan.ac.uk/researchgroups/ and search for <name of research Group>.

For information about Research generally at UCLan please go to http://www.uclan.ac.uk/research/

All outputs in CLoK are protected by Intellectual Property Rights law, including Copyright law. Copyright, IPR and Moral Rights for the works on this site are retained by the individual authors and/or other copyright owners. Terms and conditions for use of this material are defined in the policies page. 
Publication Information: Hong, N., Zhan, J., Wang, X., Stec, A.A., Richard Hull, T., Ge, H., Xing, W., Song, L., Hu, Y., Enhanced mechanical, thermal and flame retardant properties by combining graphene nanosheets and metal hydroxide nanorods for Acrylonitrile-Butadiene-Styrene copolymer composite. Composites Part A Appl Sci Manuf 64, 203-210, (2014).

\section{Enhanced mechanical, thermal and flame retardant properties by}

\section{combining graphene nanosheets and metal hydroxide nanorods}

\section{for Acrylonitrile-Butadiene-Styrene copolymer composite}

Ningning Hong ${ }^{1}$, Jing Zhan ${ }^{1}$, Xin Wang ${ }^{1}$, Anna A. Stec ${ }^{2}$, T. Richard Hull ${ }^{2}$, Hua Ge${ }^{1}$, Weiyi Xing ${ }^{1}$, Lei Song ${ }^{1 *}$, Yuan $\mathrm{Hu}^{1 *}$

1. State Key Laboratory of Fire Science, University of Science and Technology of China, Hefei, Anhui 230026, PR China

2. Centre for Fire and Hazards Science, University of Central Lancashire, Preston, PR1 2HE, UK

\section{Abstract}

Three metal hydroxide nanorods (MHR) with uniform diameters were synthesized, and then combined with graphene nanosheets (GNS) to prepare acrylonitrile-butadiene-styrene (ABS) copolymer composites. An excellent dispersion of exfoliated two-dimensional (2-D) GNS and 1-D MHR in the ABS matrix was achieved. The effects of combined GNS and MHR on the mechanical, thermal and flame retardant properties of the ABS composites were investigated. With the addition of $2 \mathrm{wt} \% \mathrm{GNS}$ and $4 \mathrm{wt} \% \mathrm{Co}(\mathrm{OH})_{2}$, the tensile strength, bending strength and storage modulus of the ABS composites were increased by $45.1 \%$, $40.5 \%$ and $42.3 \%$ respectively. The $A B S / G N S / C o(O H)_{2}$ ternary composite shows the lowest maximum weight loss rate and highest residue yield. Noticeable reduction in the flammability was achieved with the addition of GNS and $\mathrm{Co}(\mathrm{OH})_{2}$, due to the formation of more continuous and compact charred layers that retarded the mass and heat transfer between the flame and the polymer matrix.

Keywords: A. Layered structures; A. Polymer-matrix composites (PMCs); B. Mechanical properties; B. Thermal properties.

\footnotetext{
* Corresponding author. Tel/Fax: +8655163601664.

E-mail address: leisong@ustc.edu.cn (L. Song), yuanhu@ustc.edu.cn (Y. Hu)
} 


\section{Introduction}

Polymer nanocomposites have aroused tremendous interest in material science because of their unexpected properties including superior mechanical, thermal, and electronic properties relative to the traditional microcomposites [1]. Among all the nano-additives, layered nanofillers such as silicate clay (MMT), layered double hydroxide (LDH), and layered salt phosphate, etc., have been proven to impart effective reinforcement and flame retardancy with polymers owing to their lamellar structure and high aspect ratio [2-4].

Recently, another two-dimensional (2-D) nanostructure, graphene nanosheets (GNS), comprised of $\mathrm{sp}^{2}$ hybridized carbon, has been a focal point for research, due to its unique properties and potential applications. Due to the ultrahigh mechanical strength, specific surface area, and large aspect ratio, GNS are anticipated to exhibit better enhancement in mechanical strength and flame retardancy for polymer composites than MMT or LDH [5-7]. When GNS are uniformly dispersed in polymer matrices, the load transfer from the matrices to graphene is realized, which affords high mechanical strength for the polymer/GNS nanocomposites. GNS have been widely used to reinforce poly(vinyl alcohol) and impressive $150 \%$ and $1000 \%$ increases in tensile strength and Young's modulus respectively were achieved at only 1.8 vol\% GNS loading [8]. The effect of GNS on the mechanical properties of thermoset epoxy resin was also investigated and a percolation threshold value of $0.6 \mathrm{wt} \%$ was observed [9]. According to recent reports, GNS show great potential as a flame retardant additive to endow polymers with high flame retarding. Pioneering work by Huang's group has demonstrated the effectiveness of GNS as flame retardant in poly(vinyl alcohol). With the incorporation of $3 \mathrm{wt} \% \mathrm{GNS}$, the peak heat release rate (PHRR) of the composites was reduced by $49.1 \%$ due to the formation of a compact and dense char [10]. The inclusion of GNS led to an increase in both the thermal stability and flame retardancy of polystyrene, with the optimal properties obtained at 5 wt\% GNS content [11]. The flame retardant mechanism was attributed to the layered structures of GNS that acted as barriers to slow down the heat release.

Two important issues need to be addressed when both the mechanical and flammability properties of graphene-based composites are taken into account. Pristine GNS are apt to agglomerate or even restack in matrices due to the strong van der Waals force and $\pi-\pi$ interactions between the graphene sheets $[12,13]$. In addition, the improvement especially in flame retardancy, is only achieved at high GNS concentrations, which inevitably causes a reduction in the mechanical properties $[10,14]$.

To solve the above problems, many strategies have been adopted. The idea of using two or more components to achieve synergistic effect has already been explored before. In flame retardant fields, the combination of nanofillers with other flame retardants has been demonstrated to efficiently impact the mechanical and flame retardant performance of polymers [15-18]. Fang's group has revealed that the incorporation of 2-D clay or GNS and 1-D carbon nanotubes (CNT) gave rise to a sharp increase in the mechanical properties and obviously slowed down the whole combustion process $[17,18]$. Uniform exfoliation of 2-D and 1-D nanofillers and the formation of a 3-D network structures were responsible for the 
dramatic synergies. Very recently, the combination of transition metals Lewis acids and 2-D clay provided a promising method, which involved catalytically enhanced char formation and the formation of a surface-protective layer having a network structure [19, 20]. Systematic work done by Tang's group has demonstrated that dramatically improved fire retardancy of polyolefin was realized through promoting carbonization by the combination of 2-D clay with $\mathrm{Ni}_{2} \mathrm{O}_{3}[21,22]$.

In this work, a new strategy is designed in order to simultaneously improve the mechanical properties and flame retardancy of acrylonitrile-butadiene-styrene (ABS) by combining 2-D GNS and 1-D metal hydroxide nanorods (MHR). The structures and morphologies of the GNS and MHR were characterized by X-ray diffraction (XRD) and transmission electron microscopy (TEM). The mechanical properties of the resultant ABS composites were measured by the tensile and bending testing, and the dynamic mechanical analysis (DMA). Also, the thermal degradation and the flame retardant behaviors of the resultant $A B S$ composites were evaluated using thermogravimetric analysis (TGA) and cone calorimeter methods.

\section{Experimental}

\subsection{Materials}

Natural graphite (Spectrum Pure) and acetone were bought from Sinopharm Chemical Reagent Co., Ltd (Shanghai, China). Potassium permanganate $\left(\mathrm{KMnO}_{4}\right)$, sodium nitrate $\left(\mathrm{NaNO}_{3}\right)$, sulfuric acid $\left(\mathrm{H}_{2} \mathrm{SO}_{4}, 98 \%\right)$, hydrogen peroxide $\left(\mathrm{H}_{2} \mathrm{O}_{2}, 30 \%\right)$, hydrochloric acid $(\mathrm{HCl}$, $37 \%)$ and hydrazine monohydrate $\left(\mathrm{N}_{2} \mathrm{H}_{4} \cdot \mathrm{H}_{2} \mathrm{O}\right)$ were bought from Guangfu Fine Chemial Research Institute (Tianjin, China). Cobalt chloride hexahydrate $\left(\mathrm{CoCl}_{2} \cdot 6 \mathrm{H}_{2} \mathrm{O}\right)$, nickel sulfate hexahydate $\left(\mathrm{NiSO}_{4} \cdot 6 \mathrm{H}_{2} \mathrm{O}\right)$, ferric nitrate nonahydrate $\left(\mathrm{Fe}\left(\mathrm{NO}_{3}\right)_{3} \cdot 9 \mathrm{H}_{2} \mathrm{O}\right)$, urea, sodium hydroxide $(\mathrm{NaOH})$ and potassium hydroxide $(\mathrm{KOH})$ were analytical grade and purchased from Sinopharm Chemical Reagent Co., Ltd (China). ABS (727) was supplied as pellets by Qimei New Material Co., Ltd. (China).

\subsection{Synthesis of GNS and MHR}

Graphite oxide (GO) was prepared from exfoliation of natural graphite by the modified Hummers' method [23]. GO was first subjected to ultrasonication in a $500 \mathrm{~mL}$ three-neck-flask to obtain a stable suspension $(2 \mathrm{mg} / \mathrm{mL})$. Then $12 \mathrm{~mL} \mathrm{~N} \mathrm{~N}_{4} \cdot \mathrm{H}_{2} \mathrm{O}$ was added

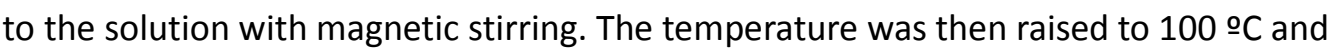
maintained for $2 \mathrm{~h}$. During the reaction process, the yellow suspension gradually changed into a black precipitate. The mixture was filtered and rinsed with distilled water, and then dried at $80^{\circ} \mathrm{C}$.

Three different MHR were synthesized by a simple hydrothermal method. The molar ratio of starting ions, the temperature and the reaction time play a critical role in the morphologies of the resultant products. In a typical synthesis of $\mathrm{Co}(\mathrm{OH})_{2}, 5 \mathrm{mmol} \mathrm{CoCl} \cdot 6 \mathrm{H}_{2} \mathrm{O}$ and $10 \mathrm{mmol}$ urea were dissolved in $50 \mathrm{~mL}$ distilled water with magnetic stirring. After stirring for $30 \mathrm{~min}$, the solution was sealed into a $60 \mathrm{~mL}$ Teflon-lined stainless steel autoclave, which was then

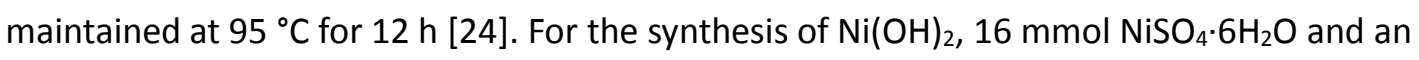


appropriate amount of $\mathrm{NaOH}$ were each dissolved in $20 \mathrm{~mL}$ deionized water. Afterwards, the latter solution was added dropwise to the former solution under vigorous stirring. The mixed solution was then transferred to an autoclave and kept in an oven maintained at $180{ }^{\circ} \mathrm{C}$ for $24 \mathrm{~h}$ [25]. The synthesis procedure of $\mathrm{FeOOH}$ was similar to that of $\mathrm{Ni}(\mathrm{OH})_{2}$, using $\mathrm{Fe}\left(\mathrm{NO}_{3}\right)_{3} \cdot 9 \mathrm{H}_{2} \mathrm{O}(12 \mathrm{mmol})$ and $\mathrm{KOH}(48 \mathrm{mmol})$ as starting materials. The hydrothermal reaction was carrried out at $100{ }^{\circ} \mathrm{C}$ for $12 \mathrm{~h}$. After being cooled to room temperature, the precipitates were filtered, washed with distilled water and ethanol, and then dried at $70{ }^{\circ} \mathrm{C}$ [26].

\subsection{Preparation of ABS/GNS/MHR nanocomposites}

The solubility of GNS in certain solvents provides a good approach to the preparation of polymer/GNS nanocomposites. GNS and/or MHR with certain proportions were dispersed to $300 \mathrm{~mL}$ acetone by sonication for $30 \mathrm{~min}$. Then ABS pellets were added to the solutions by vigorous stirring. The resulting suspension was placed in an oil-bath at $70^{\circ} \mathrm{C}$ to allow sufficient dissolution. The mixture was drop-cast into a Teflon tray and dried at $80^{\circ} \mathrm{C}$ in a vacuum oven. Finally, the ABS nanocomposites were hot pressed at $205^{\circ} \mathrm{C}$ under $10 \mathrm{MPa}$ using a Shanghai Ximaweili press vulcanizer (China). The compositions of the sample are listed in Table 1.

Table 1 Compositions of ABS nanocomposites and their corresponding mechanical properties.

\begin{tabular}{|c|c|c|c|c|c|c|c|c|c|}
\hline \multirow[t]{2}{*}{ Sample } & \multicolumn{5}{|c|}{ Compositions } & \multirow{2}{*}{$\begin{array}{l}\text { Tensile } \\
\text { Strength } \\
\text { (MPa) }\end{array}$} & \multirow{2}{*}{$\begin{array}{l}\text { Elongation } \\
\text { at break } \\
\text { (\%) }\end{array}$} & \multirow{2}{*}{$\begin{array}{l}\text { Bending } \\
\text { Strength } \\
(\mathrm{MPa})\end{array}$} & \multirow{2}{*}{$\begin{array}{l}\text { Bending } \\
\text { Modulus } \\
(\mathrm{MPa})\end{array}$} \\
\hline & $\begin{array}{l}\text { ABS } \\
\text { (wt\%) }\end{array}$ & $\begin{array}{l}\text { GNS } \\
\text { (wt\%) }\end{array}$ & $\begin{array}{l}\mathrm{Co}(\mathrm{OH})_{2} \\
\text { (wt\%) }\end{array}$ & $\begin{array}{l}\mathrm{Ni}(\mathrm{OH})_{2} \\
(\mathrm{wt} \%)\end{array}$ & $\begin{array}{l}\mathrm{FeOOH} \\
\text { (wt\%) }\end{array}$ & & & & \\
\hline ABS & 100 & & & & & $28.8 \pm 0.9$ & $28.0 \pm 2.0$ & $39.0 \pm 1.8$ & $1370 \pm 68$ \\
\hline ABSG1 & 99 & 1 & & & & $29.5 \pm 1.1$ & $4.0 \pm 0.3$ & $41.2 \pm 2.9$ & $1579 \pm 75$ \\
\hline ABSG2 & 98 & 2 & & & & $40.7 \pm 2.0$ & $3.1 \pm 0.2$ & $44.0 \pm 2.1$ & $2029 \pm 98$ \\
\hline ABSCo4 & 96 & & 4 & & & $39.1 \pm 1.6$ & $1.1 \pm 0.1$ & $45.5 \pm 1.8$ & $1494 \pm 78$ \\
\hline ABSG1Co4 & 95 & 1 & 4 & & & $43.2 \pm 2.3$ & $5.9 \pm 0.4$ & $48.4 \pm 2.1$ & $2107 \pm 86$ \\
\hline ABSG2Co4 & 94 & 2 & 4 & & & $41.8 \pm 1.7$ & $3.7 \pm 0.3$ & $54.8 \pm 3.3$ & $2253 \pm 103$ \\
\hline ABSG2Ni4 & 94 & 2 & & 4 & & $39.3 \pm 1.4$ & $2.5 \pm 0.2$ & $51.9 \pm 2.6$ & $2193 \pm 118$ \\
\hline ABSG2Fe4 & 94 & 2 & & & 4 & $40.5 \pm 1.6$ & $5.6 \pm 0.4$ & $42.7 \pm 2.3$ & $2020 \pm 92$ \\
\hline
\end{tabular}

\subsection{Measurements and Characterization}

X-ray diffraction (XRD) patterns were performed with a Japan Rigaku D/Max-Ra rotating anode X-ray diffractometer equipped with a Cu-K $\alpha$ tube and Ni filter $(\lambda=0.1542 \mathrm{~nm})$. 
Transmission electron microscopy (TEM) was used to observe the morphology with an acceleration voltage of $200 \mathrm{kV}$. Specimens for TEM observation were prepared by dispersing the sample suspension onto a lacy carbon film supported by a $\mathrm{Cu}$ grid.

Scanning electron microscopy (SEM) was performed on a XL-30 scanning electron microscope with the acceleration voltage of $20 \mathrm{kV}$. The fracture surfaces and char residues of samples were previously coated with a conductive layer of gold before analysis.

The tensile strength of the nanocomposites was measured with an electronic universal testing instrument (MTS System Co., Ltd, China) at a crosshead speed of $50 \mathrm{~mm} / \mathrm{min}$. The elongation was in the range of error, so only the tensile strength was reported.

The bending properties of the nanocomposites were tested with an electronic universal testing instrument (MTS System Co., Ltd, China) at a crosshead speed of $2 \mathrm{~mm} / \mathrm{min}$ according to GB/T8812.1-2007.

Dynamic mechanical analysis (DMA) was performed on the Perkin-Elmer Pyris Diamond DMA from $20^{\circ} \mathrm{C}$ to $170^{\circ} \mathrm{C}$ at a heating rate of $5^{\circ} \mathrm{C} / \mathrm{min}$, at a frequency of $10 \mathrm{~Hz}$ in the tensile configuration.

The thermogravimetric analysis (TGA) was carried out on the TGA Q5000 IR thermogravimetric analyzer (TA instruments) using a heating rate of $20^{\circ} \mathrm{C} / \mathrm{min}$ in a nitrogen or air atmosphere.

The combustion property of the nanocomposites was investigated using a FTT cone calorimeter according to ISO 5660 standard. Every sample with a size of $100 * 100 * 3 \mathrm{~mm}^{3}$ was heated at a heat flux of $35 \mathrm{~kW} / \mathrm{m}^{2}$.

\section{Results and Discussion}

\subsection{Characterization of GNS and MHR}

The morphologies of the as-synthesized GNS and MHR were observed by TEM. Fig. 1a shows the existence of thin carbon sheets of size up to $1 \mu \mathrm{m}$. GNS sheets seem to associate closely with each other to form an overlapped structure. As shown in Fig. 1b, the sample consists of a large quantity of wire-like $\mathrm{Co}(\mathrm{OH})_{2}$ with a uniform diameter of 40-60 nm and length of several micrometers. From Fig. 1c, the rod-like morphology is widely observed with uniform diameter and well dispersion. Based on the mutability of $\mathrm{Ni}(\mathrm{OH})_{2}$, the morphology of the final product has been successfully controlled by tuning the key parameters of molar ratio, temperature and time. FeOOH exhibits almost rod-like morphology with diameter of 80-100 nm, indicating high yield and good uniformity were achieved by the hydrothermal method (Fig. 1d).

The crystalline phases of the GO, GNS and MHR were characterized by XRD as presented in Fig. 2a. After chemical reduction, a broad (002) diffraction halo at about $24.5^{\circ}$ is observed for the GNS, corresponding to a d-spacing of $0.35 \mathrm{~nm}$. This result indicates the $\mathrm{GO}$ is well reduced and the as-reduced GNS sheets appear to partially restack [27]. In the case of $\mathrm{Co}(\mathrm{OH})_{2}$ nanorods, all the diffraction peaks can be well indexed, which are consistent with the result in the literature [24]. No peaks for other phases are found in the as-synthesized 
nanorods. $\mathrm{Ni}(\mathrm{OH})_{2}$ obtained herein has very high peak intensity, suggesting that the nanomaterials are well crystallized [25]. Pure $\mathrm{FeOOH}$ with high crystallinity has been successfully synthesized, as evidenced by the diffraction peaks attributed to orthorhombic $\alpha$-FeOOH [26]. Fig. 2b shows the TGA curves of GNS and MHR in air. There are three major weight loss steps for $\mathrm{GO}$ as the temperature increases. The first step before $100{ }^{\circ} \mathrm{C}$ is ascribed to the removal of absorbed water; the second one occurs at around $200^{\circ} \mathrm{C}$ due to the decomposition of labile oxygen functional groups; almost all the sample is oxidized in the last step when the temperature raised to $800^{\circ} \mathrm{C}$ [28]. GNS presents a gradual weight loss with an increase in temperature, which can be attributed to the removal of water molecules and labile oxygen-containing groups on the GNS sheets. A large weight loss of over $80 \mathrm{wt} \%$ occurs in the temperature range of $550-800{ }^{\circ} \mathrm{C}$ due to the oxidation of the graphene skeleton in air [27]. There is an abrupt weight loss between 250 and $350^{\circ} \mathrm{C}$ due to removal of structural water and transformation of $\mathrm{Co}(\mathrm{OH})_{2}$ to $\mathrm{Co}_{3} \mathrm{O}_{4}$. The temperature of maximum decomposition rate $\left(T_{\max }\right)$ of $296{ }^{\circ} \mathrm{C}$ is observed in the DTG curve. It is observed that the temperature for $5 \%$ weight loss $\left(\mathrm{T}_{-5 \%}\right)$ and $\mathrm{T}_{\max 1}$ of $\mathrm{Ni}(\mathrm{OH})_{2}$ shift to higher temperatures of 274.8 and $341.0^{\circ} \mathrm{C}$ respectively. The weight loss in the first step can be attributed to the loss of water and the phase transformation of $\mathrm{Ni}(\mathrm{OH})_{2}$ to $\mathrm{NiO}$. The decomposition behaviour of $\mathrm{FeOOH}$ is similar to that of $\mathrm{Co}(\mathrm{OH})_{2}$, exhibiting the $\mathrm{T}_{-5 \%}\left(261{ }^{\circ} \mathrm{C}\right)$, $\mathrm{T}_{\max }\left(274^{\circ} \mathrm{C}\right)$, and $87 \mathrm{wt} \%$ residue at $800^{\circ} \mathrm{C}$.
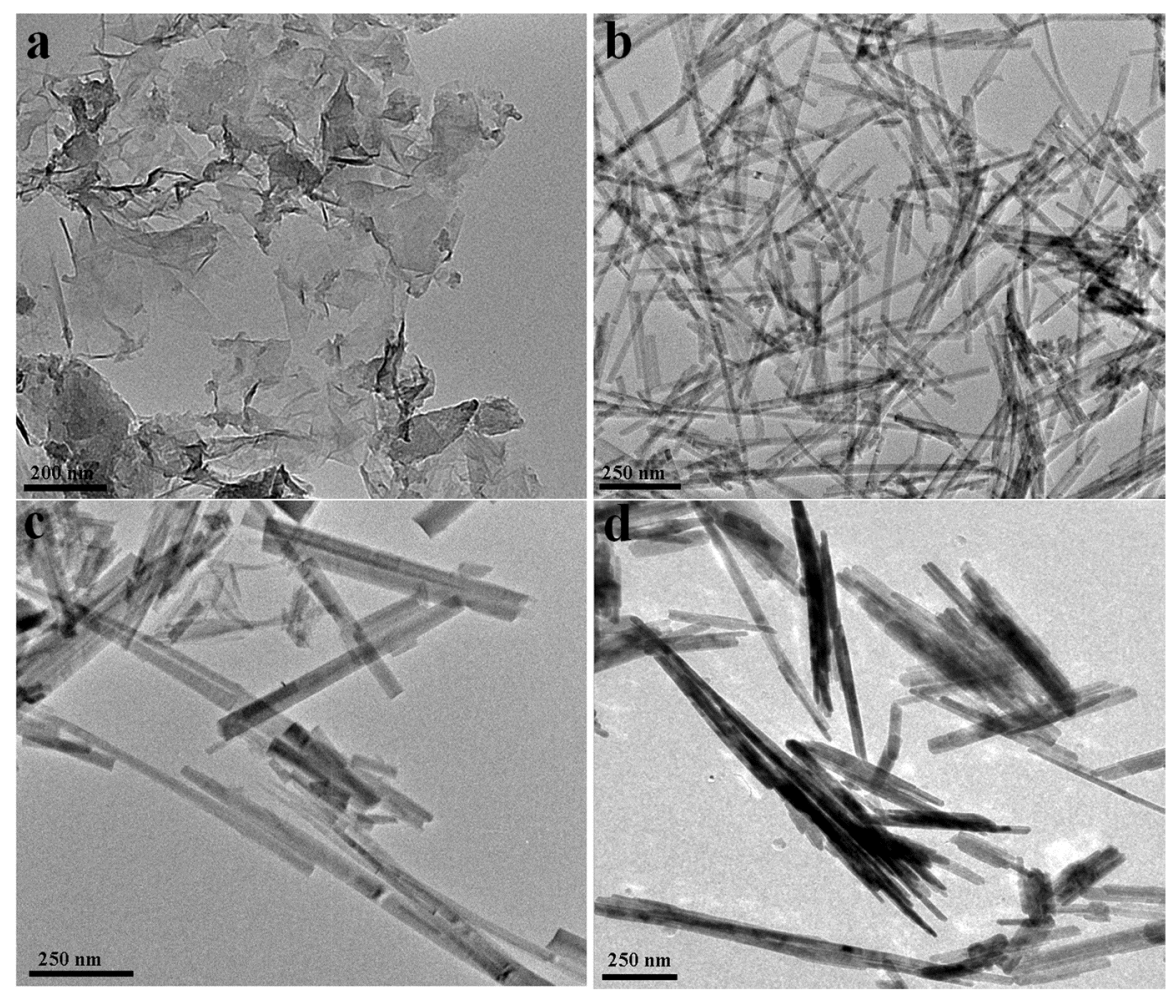

Fig. 1. TEM images of the as-prepared GNS (a), $\mathrm{Co}(\mathrm{OH})_{2}(\mathrm{~b}), \mathrm{Ni}(\mathrm{OH})_{2}(\mathrm{c})$ and $\mathrm{FeOOH}(\mathrm{d})$. 

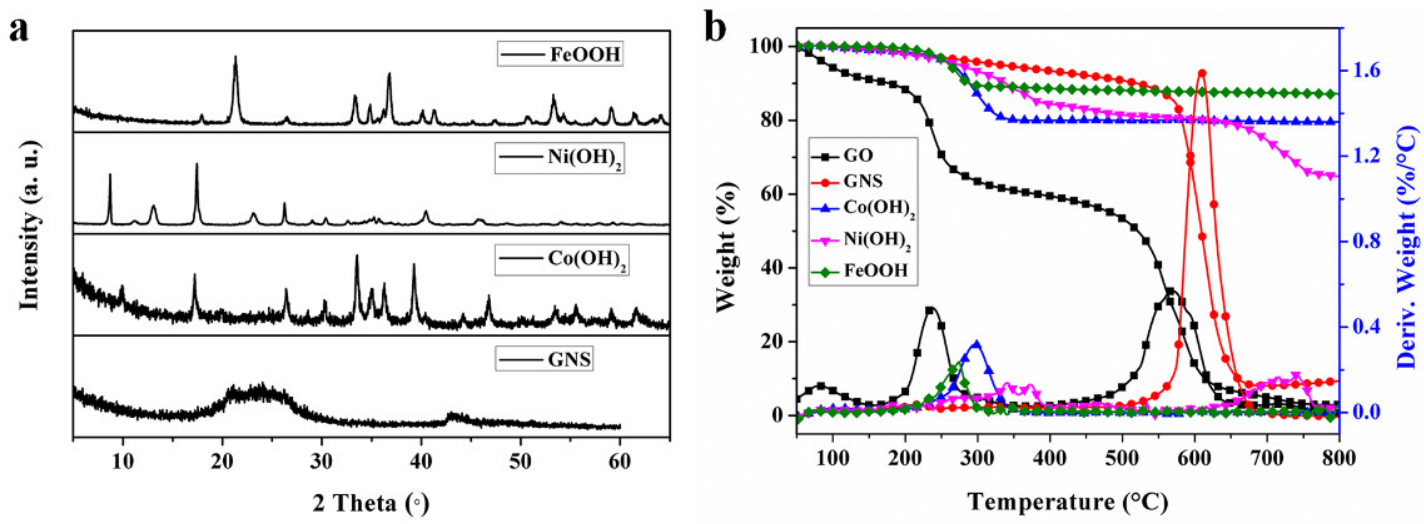

Fig. 2. XRD patterns (a) and TGA curves (b) of the $\mathrm{GNS}, \mathrm{Co}(\mathrm{OH})_{2}, \mathrm{Ni}(\mathrm{OH})_{2}$ and $\mathrm{FeOOH}$.

\subsection{Dispersion of GNS and MHR}

The dispersion state of nanofillers in a matrix is critical to the mechanical, thermal, and other properties of the composite. To investigate the morphological features of GNS and/or MHR in the ABS matrix, SEM and TEM were used. Pure ABS represents a smooth fracture surface, indicative of a typical brittle failure feature (not shown). With the introduction of 2 wt\% GNS, the surface of ABS composite becomes rough (Fig. 3a). In addition, graphene aggregates are sometimes seen on the surface due to the strong van der Waals forces between GNS sheets (marked by black circles). As revealed in Fig. 3b, the fractured surface contains randomly dispersed nanorods without visible agglomerates, suggesting good dispersion of $\mathrm{Co}(\mathrm{OH})_{2}$ in the matrix (marked by black rectangles). The ABSG2Co4 composite shows good dispersion and homogeneity on the fractured surface (Fig. 3c). 2-D GNS is embedded in and 1-D Co(OH $)_{2}$ is held tightly by the matrix, suggesting the formation of 3-D network structure. In the TEM image of ABSG2Co4 (Fig. 3d), the gray background represents the ABS matrix, while the black lines and flakes correspond to different orientations of GNS sheets (marked by black circles and rectangles). As shown in Fig. 3e and f, ABS composites with $\mathrm{Ni}(\mathrm{OH})_{2}$ and $\mathrm{FeOOH}$ exhibit almost the similar features except for rougher surfaces. No GNS aggregates could be observed in all the ternary composite, indicating that the presence of MHR could prevent the GNS from re-stacking and thus have a good dispersion in the matrix. 

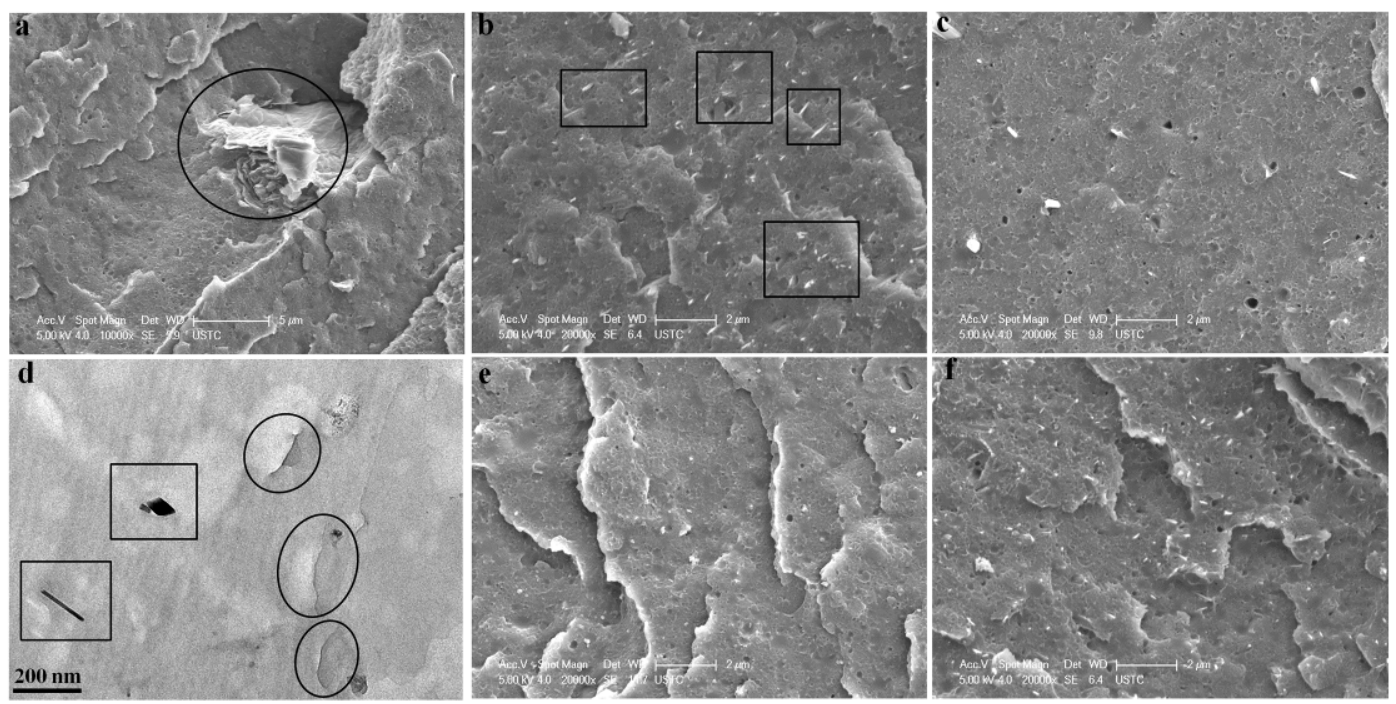

Fig. 3. SEM images of the fractured surfaces of ABSG2 (a), ABSCo4 (b), ABSG2Co4 (c), ABSG2Ni4 (e) and ABSG2Fe4 (f); TEM image of ABSG2Co4 (d).

\subsection{Mechanical properties of ABS nanocomposites}

The effects of GNS and MHR on the mechanical properties of the ABS composites are studied, with the results summarized in Table 1. Because of the high mechanical modulus of GNS, the tensile strength (TS) increases from 28.84 MPa for pure ABS to $40.71 \mathrm{MPa}$ for ABSG2, but its elongation at break largely reduces. The incorporation of $4 \mathrm{wt} \% \mathrm{Co}(\mathrm{OH})_{2}$ results in a $35.6 \%$ increase in TS, compared to pure ABS owing to the homogeneous dispersion. When the combination of GNS with $\mathrm{Co}(\mathrm{OH})_{2}$ is applied, further enhancement in the TS of the ternary composite are observed. The TS of ABS composites reaches a maximum value of $43.16 \mathrm{MPa}$ when $1 \mathrm{wt} \% \mathrm{GNS}$ and $4 \mathrm{wt} \% \mathrm{Co}(\mathrm{OH})_{2}$ are added, corresponding to an increase of $49.7 \%$. Such enhancement is attributed to the 3-D network structure formed by homogeneous dispersion of GNS and $\mathrm{Co}(\mathrm{OH})_{2}$, which facilitates the successful load transfer from the matrix to the nanofillers. The enhancement in the TS is more significant than the values reported by the previous literatures $[29,30]$. According to Tang's work, the incorporation of carbon nanotubes slightly improves the TS of linear low density polyethylene/ $\mathrm{Ni}_{2} \mathrm{O}_{3}$ compared with those of binary composites [29].An increase in mechanical strength was reported for epoxy/graphene nanoplate/carbon nanotube composites, where only $14.5 \%$ increase in the TS was obtained compared to epoxy/graphene nanoplate composites [30]. Compared to the ABSG2Co4, ABSG2Ni4 and ABSG2Fe4 show slightly reduced TS of 39.29 and $40.46 \mathrm{MPa}$, respectively, due to the decreased aspect ratio of $\mathrm{Ni}(\mathrm{OH}) 2$ and $\mathrm{FeOOH}$ nanorods. The formation of a network structure due to the homogeneous dispersion of two types of nanofillers is responsible for the improvement in the mechanical properties of the composites.

From Table 1, it can be also seen that the incorporation of GNS significantly increases bending strength (BS) and bending modulus (BM) of the ABS composites, due to the high intrinsic strength and modulus of GNS [31]. The inclusion of $4 \mathrm{wt} \% \mathrm{Co}(\mathrm{OH})_{2}$ also slightly 
improves the ultimate $\mathrm{BS}$ and $\mathrm{BM}$ of the $\mathrm{ABS}$ composite owing to the reinforced effect derived from the large aspect ratio of the nanorod. With the simultaneous addition of GNS and $\mathrm{Co}(\mathrm{OH})_{2}$, it is found that both $\mathrm{BS}$ and $\mathrm{BM}$ are further increased to $54.8 \mathrm{MPa}$ and 2253 $\mathrm{MPa}$, respectively. In comparison with $\mathrm{Ni}(\mathrm{OH})_{2}$ and $\mathrm{FeOOH}, \mathrm{Co}(\mathrm{OH})_{2}$ shows more obvious synergistic effect with GNS on improving the BS and BM of the composites.

The mechanical reinforcement is also reflected on the dynamic mechanical property. The storage modulus ( $E^{\prime}$ ) as a function of temperature curves were plotted in Fig. 4 for ABS and its composites. The incorporation of GNS into ABS gives rise to a noticeable increase in $E^{\prime}$ of the composites due to the rigid nature of GNS. For instance, $E^{\prime}$ value at $30^{\circ} \mathrm{C}$ in the glassy region is $1796 \mathrm{MPa}$, yielding $28.7 \%$ increment compared to pure ABS (1396 MPa). The incorporation of $4 \mathrm{wt} \% \mathrm{Co}(\mathrm{OH})_{2}$ gives rise to a reduced $\mathrm{E}^{\prime}$ value of the composite. A combination effect of GNS and $\mathrm{Co}(\mathrm{OH})_{2}$ on the $E^{\prime}$ value of the composites in the glassy region is clearly observed. The $E^{\prime}$ values of the ABSG1Co4 and ABSG2Co4 composites at $30{ }^{\circ} \mathrm{C}$ are 2110 and $1987 \mathrm{MPa}$, respectively, which are $51.1 \%$ and $42.3 \%$ higher than that of pure ABS. This phenomenon can be attributed to the better dispersion and improved interfacial interaction between GNS and ABS matrix due to the existence of $\mathrm{Co}(\mathrm{OH})_{2}$ nanorods. By comparison, the reinforcement effects are not observed in ABSG2Ni4 and ABSG2Fe4 composites, because $\mathrm{Co}(\mathrm{OH})_{2}$ nanorod with higher aspect ratio results in stronger reinforcement.

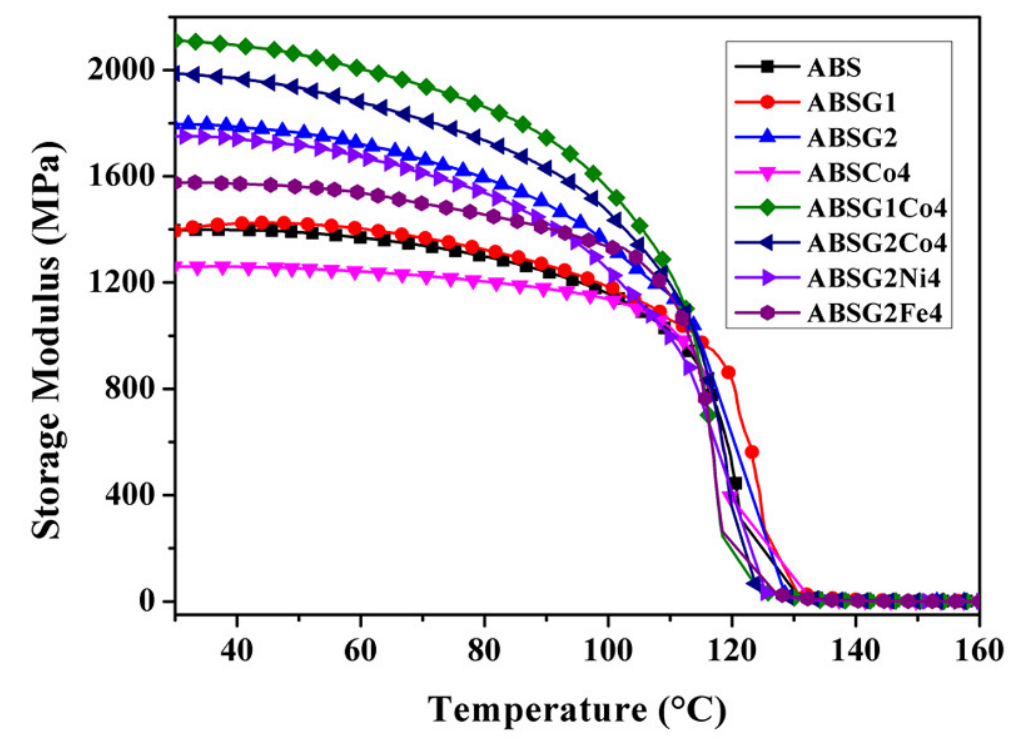

Fig. 4. Storage modulus (EO) versus temperature plots of ABS and its nanocomposites.

\subsection{Thermal stability of ABS nanocomposites}

The thermal stability of ABS and its nanocomposites was characterized by TGA under both nitrogen and air. The TGA $(a, c)$ and DTG $(b, d)$ curves are plotted in Fig. 5, and according results are summarized in Table 2 . ABS degrades with a large single peak at $382.6^{\circ} \mathrm{C}$ with little residue left, implying that pure ABS itself has weak charring ability. The addition of GNS does not visibly affect the thermal stability of the resin except for improving the residue to $3.5 \mathrm{wt} \%$. However, the addition of $\mathrm{Co}(\mathrm{OH})_{2}$ plays an important role on the 
thermal stability of the ABS composite. The $T_{-5 \%}$ and $T_{\max }$ of the ABSCo 4 composite are found to be lower than that of pure $A B S$, which could be attributed to the dehydration reaction of $\mathrm{Co}(\mathrm{OH})_{2}$ at low temperature. It is surprising that the residue is significantly increased to $13.7 \mathrm{wt} \%$. Moreover, the rate of weight loss is greatly reduced with the incorporation of $\mathrm{Co}(\mathrm{OH})_{2}$. The overall increased amount of residue may be due to the carbonization effect of $\mathrm{Co}(\mathrm{OH})_{2}$ that promotes the pyrolytic species of ABS into solid char [19]. The simultaneous addition of GNS and $\mathrm{Co}(\mathrm{OH})_{2}$ further increases the amount of residue (15.7 wt\%) and decreases the mass loss rate (33.9\%). The combination effect could be observed in the systems containing graphene and metal hydroxides, where the $T_{\max }$ and residue amount are greatly increased due to the physical barrier of graphene and the catalytic carbonization of $\mathrm{Co}(\mathrm{OH})_{2}$. As far as the residue yield is concerned, the ABSG2Ni4 and ABSG2Fe4 composites are not so efficient as ABSG2Co4, which is probably assigned to that the addition of $\mathrm{Ni}(\mathrm{OH})_{2}$ or $\mathrm{FeOOH}$ can not form an effective barrier due to lower aspect ratio.
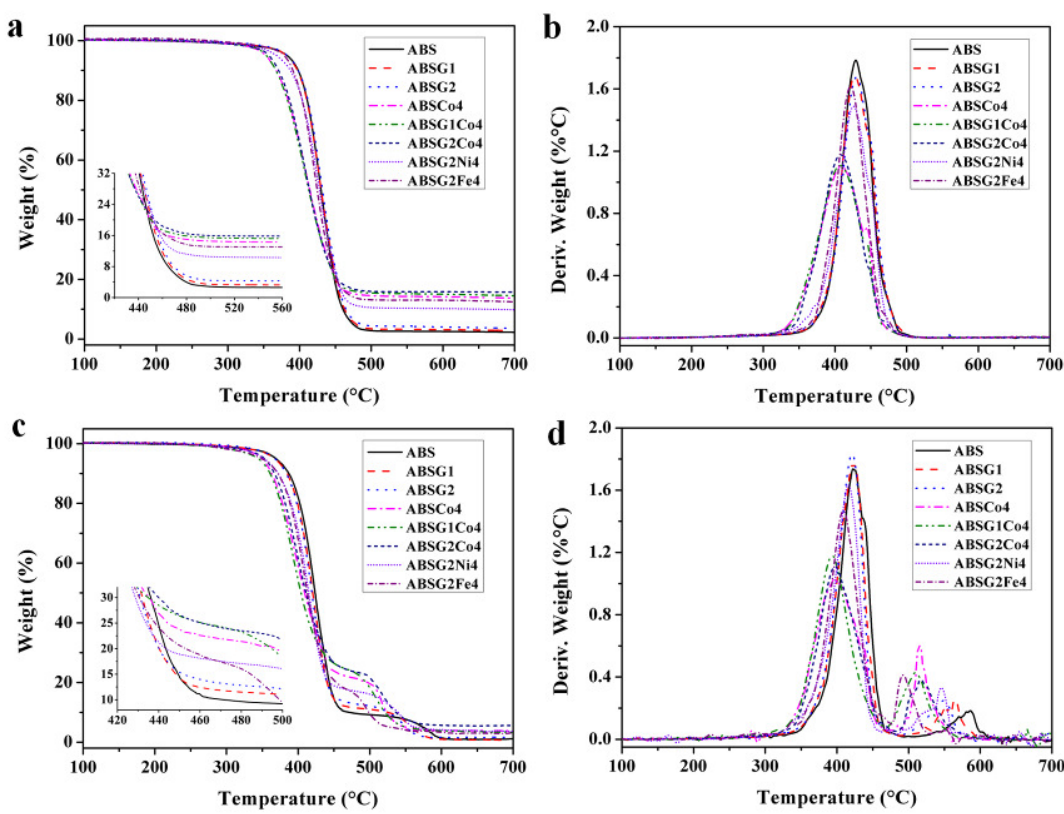

Fig. 5. TG and DTG curves of ABS and its nanocomposites in nitrogen ( $a$ and $b$ ) and air ( $c$ and d) atmosphere. Insets in (a) and (c) are the enlarged areas of weight loss. 
Table 2 TGA data of ABS and its nanocomposites in both nitrogen and air atmosphere.

\begin{tabular}{|c|c|c|c|c|c|c|c|c|}
\hline \multirow[t]{2}{*}{ Sample- } & \multicolumn{2}{|c|}{$\mathrm{T}_{-5 \%}(\stackrel{\circ}{ } \mathrm{C})$} & \multicolumn{2}{|c|}{$\mathrm{T}_{\max 1}(\stackrel{\circ}{ } \mathrm{C})$} & \multicolumn{2}{|c|}{$\mathrm{T}_{\max 2}(\stackrel{\circ}{ } \mathrm{C})$} & \multicolumn{2}{|c|}{$\begin{array}{l}\text { Residue at } 700 \circ \mathrm{C} \\
\text { (wt\%) }\end{array}$} \\
\hline & $\mathrm{N}_{2}$ & Air & $\mathrm{N}_{2}$ & Air & $\mathrm{N}_{2}$ & Air & $\mathrm{N}_{2}$ & Air \\
\hline ABS & 382.6 & 368.8 & 429.1 & 422.6 & - & 586.3 & 2.3 & 1.1 \\
\hline ABSG1 & 382.0 & 367.2 & 427.2 & 422.0 & - & 563.4 & 2.8 & 0.8 \\
\hline ABSG2 & 382.9 & 367.5 & 429.9 & 420.9 & - & 548.7 & 3.5 & 1.1 \\
\hline ABSCo4 & 352.9 & 349.0 & 409.0 & 395.9 & - & 514.1 & 13.7 & 3.9 \\
\hline ABSG1Co4 & 353.7 & 344.2 & 406.6 & 394.2 & - & 509.1 & 14.5 & 3.3 \\
\hline ABSG2Co4 & 357.4 & 352.7 & 407.2 & 397.3 & - & 517.0 & 15.7 & 5.6 \\
\hline ABSG2Ni4 & 368.2 & 352.0 & 425.9 & 415.9 & - & 545.9 & 9.8 & 3.6 \\
\hline ABSG2Fe4 & 378.7 & 349.5 & 421.9 & 408.0 & - & 491.3 & 12.5 & 3.1 \\
\hline
\end{tabular}

Thermal degradation of the samples in air is significantly different from that in nitrogen. ABS exhibits a two-step weight loss: the first step $\left(T_{\max 1}=422.6^{\circ} \mathrm{C}\right)$ is attributed to the molecular chain scission and the second one $\left(T_{\max 2}=586.3^{\circ} \mathrm{C}\right)$ is due to the oxidation of residual species. The $T_{-5 \%}$ and $T_{\max 1}$ of the ABS/GNS composites are seldom changed, while the $T_{\max 2}$ is visibly reduced, inferring the poor thermal oxidation resistance of GNS in air. Compared to pure $A B S$, the $T_{-5 \%}$ and $T_{\max }$ of $A B S C 04$ composite are largely shifted to lower temperatures, especially for the decrease of $\mathrm{T}_{\max 2}$ (about $70^{\circ} \mathrm{C}$ ). Interestingly, the rate of weight loss is significantly reduced by $40.7 \%$ and the final residue is increased to $3.9 \mathrm{wt} \%$. In this sense, cobalt oxide promotes char formation at an earlier temperature that decreases the evolution of pyrolytic gases through enhancing the char formation. When GNS are further added, though the $T_{-5 \%}$ and $T_{\max }$ of the ternary composite are largely decreased, the rate of mass loss is reduced most. It is worth noting that the combination of GNS and $\mathrm{Co}(\mathrm{OH})_{2}$ leads to the highest amount of residue (5.6 wt\%). In the enlarged area, the ternary composite displays a higher weight of residue than the individually filled binary composites with GNS or $\mathrm{Co}(\mathrm{OH})_{2}$. Therefore there is a combined effect in terms of thermal oxidative degradation. Similarly, the thermal stability of ABSG2N4 and ABSG2Fe4 is inferior to that of ABSG2Co4, but much superior to that of pure ABS.

\subsection{Flame retardancy of ABS nanocomposites}

The flammability of polymeric materials is commonly characterized by cone calorimeter, which is very effective to evaluate their fire properties under real world fire conditions. Fig. 6 shows the HRR versus time curves for ABS and its nanocomposites. Important parameters including time to ignition ( $\left.t_{\text {ign }}\right)$, time to PHRR ( $\left.t_{\text {PHRR }}\right)$, total heat release (THR), mean specific extinction area (mSEA), mean mass loss rate (mMLR) and fire growth index (FGI), are 
recorded in Table 3. Pristine ABS is easy to be ignited ( $\left.\mathrm{t}_{\mathrm{ign}}=49 \mathrm{~s}\right)$ and a rather sharp PHRR value $\left(843 \mathrm{~kW} / \mathrm{m}^{2}\right)$ yields after $173 \mathrm{~s}$. In comparison with $A B S$, the PHRR of the composite is slightly increased when GNS is added. The $t_{i g n}$ is shorten due to the high thermal conductivity of GNS [11]. Incorporating GNS shows little effect on the THR, mSEA and mMLR values. The inclusion of $4 \mathrm{wt} \% \mathrm{Co}(\mathrm{OH})_{2}$ gives rise to a $9.8 \%$ reduction in PHRR as compared to pure ABS. However, it exhibits weak effect on reducing THR and mSEA, but the mMLR is suppressed to an extent. For the trinary ABS nanocomposites, the PHRR displays a $30.5 \%$ reduction when $2 \mathrm{wt} \%$ GNS and $4 \mathrm{wt} \% \mathrm{Co}(\mathrm{OH})_{2}$ are combined. Moreover, there is an obvious synergistic effect between GNS and $\mathrm{CO}(\mathrm{OH})_{2}$ on reducing fire hazards of $A B S$ composite, including reduced mSEA and $\mathrm{mMLR}$, as compared to the binary ABSG2 and ABSCo 4 composites. It could be explained by the combination effect of the physical barrier of GNS that prevents the escape of volatile gases and the cartalytic carbonization of $\mathrm{Co}(\mathrm{OH})_{2}$ that favors the char formation [29]. The flame retardancy of ABSG2Ni4 and ABSG2Fe4 is also improved with PHRR to be 638 and $658 \mathrm{KW} / \mathrm{m}^{2}$, respectively. The $\mathrm{FGI}$, defined as the ratio of PHRR and $t_{P H R R}$, could be used to measure the overall fire behaviors of $A B S$ composites. The smallest value of 3.18 is observed in the ABSG2Co4 composites, demonstrating highest flame retardancy.

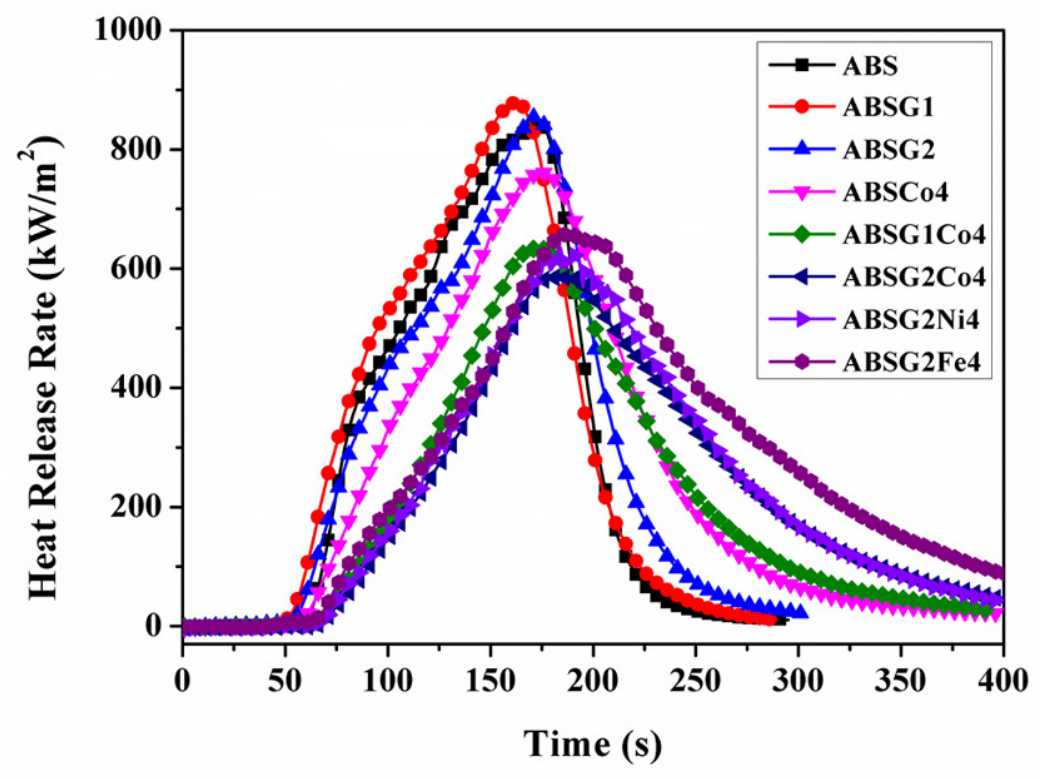

Fig. 6. Heat release rate versus time curves for ABS and its nanocomposites. 
Table 3 Cone calorimeter data of ABS and its nanocomposites at a heat flux of $35 \mathrm{~kW} / \mathrm{m}^{2}$.

\begin{tabular}{llllllll}
\hline Sample & $\mathrm{t}_{\text {ign }}(\mathrm{s})$ & $\mathrm{t}_{\text {PHRR }}(\mathrm{s})$ & $\begin{array}{l}\text { PHRR } \\
\left(\mathrm{KW} / \mathrm{m}^{2}\right)\end{array}$ & $\begin{array}{l}\mathrm{THR} \\
\left(\mathrm{MJ} / \mathrm{m}^{2}\right)\end{array}$ & $\begin{array}{l}\mathrm{mSEA} \\
\left(\mathrm{m}^{2} / \mathrm{Kg}\right)\end{array}$ & $\begin{array}{l}\mathrm{mMLR} \\
(\mathrm{Kg} / \mathrm{Kg})\end{array}$ & $\begin{array}{l}\mathrm{FGI} \\
\left(\mathrm{KW} / \mathrm{s} \cdot \mathrm{m}^{2}\right)\end{array}$ \\
\hline ABS & 49 & 173 & 843 & 84.08 & 1175 & 0.116 & 4.87 \\
ABSG1 & 35 & 164 & 878 & 85.63 & 1151 & 0.167 & 5.35 \\
ABSG2 & 34 & 171 & 855 & 89.00 & 1096 & 0.114 & 5.00 \\
ABSC04 & 46 & 175 & 760 & 93.99 & 1235 & 0.082 & 4.34 \\
ABSG1C04 & 43 & 173 & 634 & 79.30 & 1300 & 0.076 & 3.66 \\
ABSG2C04 & 45 & 184 & 586 & 88.49 & 709 & 0.067 & 3.18 \\
ABSG2Ni4 & 35 & 183 & 638 & 90.35 & 976 & 0.083 & 3.49 \\
ABSG2Fe4 & 39 & 188 & 658 & 92.29 & 1107 & 0.111 & 3.50 \\
\hline
\end{tabular}

The flame retardant efficiency has a direct relationship with the structure and yield of the char formed. Fig. 7 shows the digital photographs of the residual chars from the combustion of ABS composites. Pure ABS is almost completely burnt, and little char is obtained (Fig. 7a). The residue of ABSG2 is very loose as revealed in Fig. 7b. Layered GNS could somewhat prevent the evolution of gaseous products, but its efficiency is not high. The color of the char of ABSCO4 composite turns into black, indicating that cobalt compound promotes char formation (Fig. 7c). In the case of ABSG2Co4, the char looks more continuous and compact, which is likely to reduce the HRR (Fig.7d). Promoting char formation of ABS itself during combustion will be beneficial for reducing its flammability [32]. The combination effect of the physical barriers of GNS and the catalytic carbonization of $\mathrm{Co}(\mathrm{OH})_{2}$ is responsible for the improved char residue. In Fig.7e, the morphology of the final residues of ABSG2Ni4 seems to be similar with that of $A B S G 2 C 04$. For $A B S G 2 F e 4$ composite, the rufous and island-like residue indicates that the main component is $\mathrm{Fe}_{2} \mathrm{O}_{3}$ from the decomposition of $\mathrm{FeOOH}$ rather than amorphous carbon (Fig.7f).

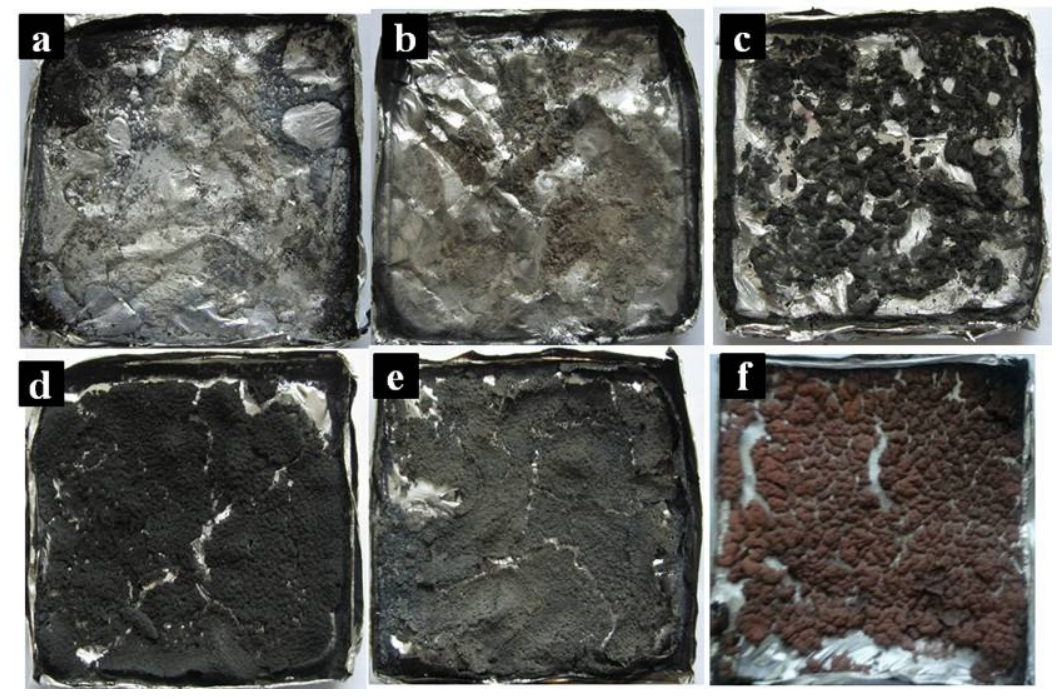

Fig. 7. Digital photographs of the char residues of ABS and its nanocomposites. 


\section{Conclusions}

The $\mathrm{Co}(\mathrm{OH})_{2}, \mathrm{Ni}(\mathrm{OH})_{2}$ and $\mathrm{FeOOH}$ nanorods were successfully synthesized by a facile hydrothermal process, and their structures were characterized by TEM, XRD, FTIR and TGA. The 1-D MHR and their combination with 2-D GNS were used to prepare ABS composites. Stacking of individual 2-D GNS sheets is somewhat hindered by introducing 1-D MHR nanorods, resulting in the formation of 3-D nanohybrids network in the ABS matrix. A combined effect exists between $\mathrm{GNS}$ and $\mathrm{Co}(\mathrm{OH})_{2}$ in the mechanical properties, thermal stability and flame retardancy of ABS composites. When 2 wt\% GNS and 4 wt\% $\mathrm{Co}(\mathrm{OH})_{2}$ were combined, the resulting composite exhibits significant mechanical improvements. The thermal stability of $A B S$ is also obviously enhanced with the addition of GNS and $\mathrm{Co}(\mathrm{OH})_{2}$. The combination of GNS and $\mathrm{Co}(\mathrm{OH})_{2}$ could confer better flame retardancy on ABS relative to all other components. A more continual, compact charred layers were formed in the ABS/GNS/Co(OH $)_{2}$ composite. This improvement is partially attributed to the formed 3-D network due to the good dispersion of GNS and $\mathrm{Co}(\mathrm{OH})_{2}$, and partially to the carbonization of degradation products of ABS catalyzed by $\mathrm{Co}(\mathrm{OH})_{2}$.

\section{Acknowledgements}

The work was financially supported by the National Key Technology R\&D Program (2013BAJ01B05), the National Natural Science Foundation of China (No.21374111), the China Postdoctoral Science Special Foundation (2013T60621) and the Institute of Nanotechnology and Bioengineering of the University of Central Lancashire in the UK.

\section{References}

[1] Kumar AP, Depan D, Tomer NS, Singh RP. Nanoscale particles for polymer degradation and stabilization-Trends and future perspectives. Prog Polym Sci 2009;34(6): 479-515.

[2] Bitinis N, Hernandez M, Verdejo R, Kenny JM, Lopez-Manchado MA. Recent Advances in Clay/Polymer Nanocomposites. Adv Mater 2011;23(44):5229-36.

[3] Leroux F, Besse JP. Polymer interleaved layered double hydroxide: A new emerging class of nanocomposites. Chem Mater 2001;13(10):3507-15.

[4] Alongi J, Frache A. Flame retardancy properties of alpha-zirconium phosphate based composites. Polym Degrad Stabil 2010;95(9):1928-33.

[5] Kotov NA. Materials science: Carbon sheet solutions. Nature 2006;442(7100):254-5.

[6] Zaman I, Kuan HC, Dai JF, Kawashima N, Michelmore A, Sovi A, Dong SY, Luong L, Ma J. From carbon nanotubes and silicate layers to graphene platelets for polymer nanocomposites. Nanoscale 2012;4(15):4578-86.

[7] Putz KW, Compton OC, Palmeri MJ, Nguyen ST, Brinson LC. High-Nanofiller-Content 
Graphene Oxide-Polymer Nanocomposites via Vacuum-Assisted Self-Assembly. Adv Funct Mater 2010;20(19):3322-9.

[8] Zhao X, Zhang QH, Chen DJ, Lu P. Enhanced Mechanical Properties of Graphene-Based Poly(vinyl alcohol) Composites. Macromolecules 2010;43(5):2357-63.

[9] Fang M, Zhang Z, Li JF, Zhang HD, Lu HB, Yang YL. Constructing hierarchically structured interphases for strong and tough epoxy nanocomposites by amine-rich graphene surfaces. J Mater Chem 2010;20(43):9635-43.

[10] Huang GB, Gao JR, Wang X, Liang HD, Ge CH. How can graphene reduce the flammability of polymer nanocomposites? Mater Lett 2012;66(1):187-9.

[11] Han YQ, Wu Y, Shen MX, Huang XL, Zhu JJ, Zhang XG. Preparation and properties of polystyrene nanocomposites with graphite oxide and graphene as flame retardants. J Mater Sci 2013;48(12):4214-22.

[12] Lin Y, Jin J, Song M. Preparation and characterisation of covalent polymer functionalized graphene oxide. J Mater Chem 2011;21(10):3455-61.

[13] Cao YW, Lai ZL, Feng JC, Wu PY. Graphene oxide sheets covalently functionalized with block copolymers via click chemistry as reinforcing fillers. J Mater Chem 2011;21(48):9271-8.

[14] Hong NN, Pan Y, Zhan J, Wang BB, Zhou KQ, Song L, et al. Fabrication of graphene/Ni-Ce mixed oxide with excellent performance for reducing fire hazard of polypropylene. Rsc Adv 2013;3(37):16440-8.

[15] Yang F, Nelson GL. Combination effect of nanoparticles with flame retardants on the flammability of nanocomposites. Polym Degrad Stabil 2011;96(3):270-6.

[16] Yu AP, Ramesh P, Sun XB, Bekyarova E, Itkis ME, Haddon RC. Enhanced Thermal Conductivity in a Hybrid Graphite Nanoplatelet-Carbon Nanotube Filler for Epoxy Composites. Adv Mater 2008;20(24):4740-4.

[17] Ma HY, Tong LF, Xu ZB, Fang ZP. Synergistic effect of carbon nanotube and clay for improving the flame retardancy of ABS resin. Nanotechnology 2007;18(37): 375602.

[18] Song PA, Liu LN, Fu SY, Yu YM, Jin CD, Wu Q, Zhang Y, Li Q. Striking multiple synergies created by combining reduced graphene oxides and carbon nanotubes for polymer nanocomposites. Nanotechnology 2013;24(12):125704.

[19] Jang J, Kim J, Bae JY. Effects of Lewis acid-type transition metal chloride additives on the thermal degradation of ABS. Polym Degrad Stabil 2005;88(2):324-32.

[20] Kashiwagi T, Danyus R, Liu MF, Zammarano M, Shields JR. Enhancement of char formation of polymer nanocomposites using a catalyst. Polym Degrad Stabil 2009;94(11):2028-35.

[21] Tang T, Chen XC, Chen H, Meng XY, Jiang ZW, Bi WG. Catalyzing carbonization of polypropylene itself by supported nickel catalyst during combustion of polypropylene/clay nanocomposite for improving fire retardancy. Chem Mater 2005;17(11):2799-802.

[22] Song RJ, Jiang ZW, Yu HO, Liu J, Zhang ZJ, Wang QW, Tang T. Strengthening carbon 
deposition of polyolefin using combined catalyst as a general method for improving fire retardancy. Macromol Rapid Comm 2008;29(10):789-93.

[23] Park S, An JH, Piner RD, Jung I, Yang DX, Velamakanni A, Nguyen ST, Ruoff RS. Aqueous Suspension and Characterization of Chemically Modified Graphene Sheets. Chem Mater 2008;20(21):6592-4.

[24] Wang $\mathrm{ZH}$, Chen $\mathrm{XY}$, Zhang $\mathrm{M}$, Qian $\mathrm{YT}$. Synthesis of $\mathrm{CO}_{3} \mathrm{O}_{4}$ nanorod bunches from a single precursor $\mathrm{CO}\left(\mathrm{CO}_{3}\right)(0.35) \mathrm{Cl}_{0.20}(\mathrm{OH})_{1.10}$. Solid State Sci 2005;7(1):13-5.

[25] Dong LH, Chu Y, Sun WD. Controllable synthesis of nickel hydroxide and porous nickel oxide nanostructures with different morphologies. Chem-Eur J 2008;14(16):5064-72.

[26] Ou P, Xu G, Ren ZH, Hou XH, Han GR. Hydrothermal synthesis and characterization of uniform alpha-FeOOH nanowires in high yield. Mater Lett 2008;62(6-7):914-7.

[27] He GY, Chen HQ, Zhu JW, Bei FL, Sun XQ, Wang X. Synthesis and characterization of graphene paper with controllable properties via chemical reduction. J Mater Chem 2011;21(38):14631-8.

[28] Dubin S, Gilje S, Wang K, Tung VC, Cha K, Hall AS, Farrar J, Varshneya R, Yang Y, Kaner RB. A One-Step, Solvothermal Reduction Method for Producing Reduced Graphene Oxide Dispersions in Organic Solvents. Acs Nano 2010;4(7):3845-52.

[29] Yu H, Liu J, Wang Z, Jiang ZW, Tang T. Combination of Carbon Nanotubes with $\mathrm{Ni}_{2} \mathrm{O}_{3}$ for Simultaneously Improving the Flame Retardancy and Mechanical Properties of Polyethylene. J Phys Chem C 2009;113(30):13092-7.

[30] Yang SY, Lin WN, Huang YL, Tien HW, Wang JY, Ma CCM, Li SM, Wang YS. Synergetic effects of graphene platelets and carbon nanotubes on the mechanical and thermal properties of epoxy composites. Carbon 2011;49(3):793-803.

[31] Farahani RD, Dalir H, Aissa B, Khakani MAE, Lévesque M, Therriault D. Micro-infiltration of three-dimensional porous networks with carbon nanotube-based nanocomposite for material design. Compos Part A-Appl S 2011;(42):1910-9.

[32] Song PA, Xu LH, Guo ZH, Zhang Y, Fang ZP. Flame-retardant-wrapped carbon nanotubes for simultaneously improving the flame retardancy and mechanical properties of polypropylene. J Mater Chem 2008;(18):5083-91. 